\title{
Data Replicas in Distributed Information Services
}

\author{
H. M. GLADNEY \\ IBM Almaden Research Center
}

In an information distribution network in which records are repeatedly read, it is cost-effective to keep read-only copies in work locations. This paper presents a method of updating replicas that need not be immediately synchronized with the source data or with each other. The method allows an arbitrary mapping from source records to replica records. It is fail-safe, maximizes workstation autonomy, and is well suited to a network with slow, unreliable, and/or expensive communications links.

The algorithm is a manipulation of queries, which are represented as short encodings. When a response is generated, we record which portion of the source database was used. Later, when the source data are updated, this information is used to identify obsolete replicas. For each workstation, the identity of obsolete replicas is saved until a workstation process asks for this information. This workstation process deletes each obsolete replica, and replaces it by an up-to-date version either promptly or the next time the application asks for this particular item. Throughout, queries are grouped so that the impact of each source update transaction takes effect atomically at each workstation.

Optimizations of the basic algorithm are outlined. These overlap change dissemination with user service, allow the mechanism to be hidden within the data delivery subsystem, and permit very large networks.

Categories and Subject Descriptors: C.2.4 [Computer-Communications Networks]: Distributed Systems-distributed applications, distributed databases; H.2.4 [Database Management]: Systemsdistributed systems, query processing

General Terms: Algorithms, Performance

Additional Key Words and Phrases: Cache, consistency, copies, currency, query, read-only, replicas, transaction, workstation

\section{INTRODUCTION}

With increasing deployment of personal computers, it is of interest to consider how best to partition applications between remote data servers and workstations. For information dissemination, it is possible to reduce the communications requirements for remote workstations and, at the same time, to improve performance over what has been previously feasible. The approach reported in this paper is particularly suited to public information systems.

\footnotetext{
Author's address: IBM Almaden Research Center, San Jose, CA 95120.

Permission to copy without fee all or part of this material is granted provided that the copies are not made or distributed for direct commercial advantage, the ACM copyright notice and the title of the publication and its date appear, and notice is given that copying is by permission of the Association for Computing Machinery. To copy otherwise, or to republish, requires a fee and/or specific. permission.
}

(C) 1989 ACM 0362-5915/89/0300-0075 $\$ 01.50$ 
A simple and flexible strategy is to store responses received in a workstation cache that is consulted before each query is forwarded to a data server. This approach accommodates varying communications media and different usage statistics at each workstation. Saved responses are data replicas that must be updated to reflect changes to their sources. We present a new update protocol. Each record transmitted to a workstation may be an arbitrary function of the source database content.

The synchronization method is for read-only data held in secondary repositories, but does not interfere with well-known methods for managing database updates and serializing concurrent transactions, and does not support dynamically generated queries. It is ideal for application environments in which most interactions only query the database, and in which updates originate in a small number of workstations. It can be optimized so that the communications traffic to manage obsolescence is low, and so that no workstation user perceives performance degradation related to database updates.

Section 2 describes the generic origin of a class of replica management problems and suggests that arbitrary mappings from source data to replica data are needed to minimize network traffic in distributed applications. It continues with an informal description of our approach. Essentially the same algorithm is formally described in Section 3, which restricts itself to the case of a single workstation accessing a single remote database. The algorithm has two parts: computation of sets of replicas that must be updated atomically, and dissemination to the workstation under the assumption that communications are unreliable. Section 4 shows how the method is applied to a public information system. A prototype implementation demonstrated that, even on quite modest processors, responsiveness was better than adequate for realistic situations. Extension of the algorithm to large networks is sketched in Section 5, which addresses hierarchies of stores and the possibility that different locations may need marginally different data views. Finally, Section 6 relates our method to earlier work on replica management, argues that the method is widely applicable, and that the burden on application programmers is modest.

The work reported is based on a portion of a prototype built by Mattson, Lorch, Reuter and the author [13], with contributions from others. All the essential elements have been exercised in test environments.

\section{OVERVIEW OF THE APPROACH TO VERSION MANAGEMENT}

\subsection{Origin of the Problem}

As a starting point, consider a system with "dumb" terminals attached to a computer containing a source database. Each interactive session may invoke several presentation subroutines (see Figure 1). Each presentation generator prepares the variable part of the screens of a single schema; each presentation value is a function solely of the arguments to the presentation generator and the current state of a database. The programs are general to applications in retail sales, public expositions, and guided education; application specifics, including the links for navigation among screens, are captured in databases that represent directed graphs and entity-attribute relations. These data may be implemented ACM Transactions on Database Systems, Vol. 14, No. 1, March 1989. 


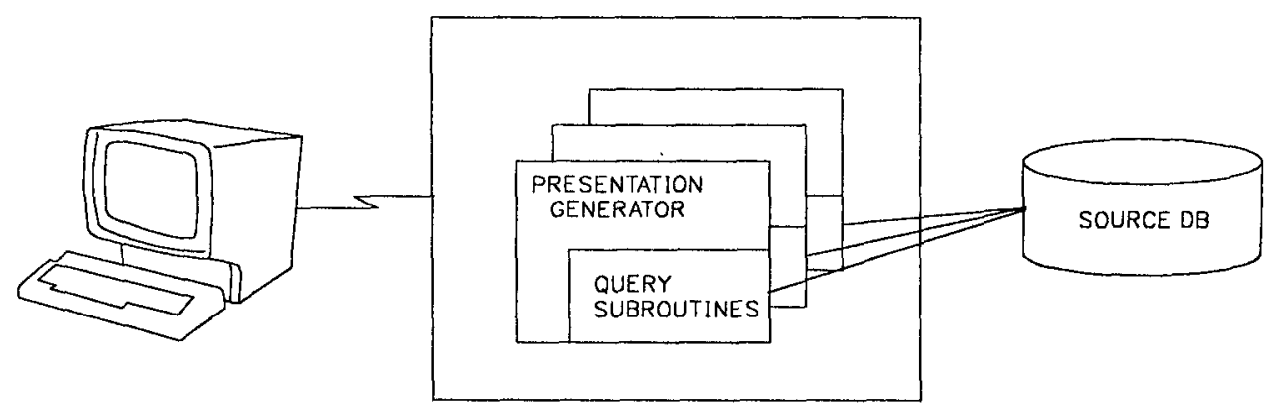

Fig. 1. Simple configuration for a single user.

in any mixture of representations-flat files, relations, hierarchies, etc. Representational detail is masked by the query subroutines.

For a remote workstation, the application may be partitioned (see Figure 2) between the workstation and a data server. The terminal management portion of each presentation generator becomes a presentation manager. The query subroutines become response generators to which presentation managers in the workstation direct requests. The shell program in the data server directs each request to the correct response generator.

Details of the service split between the work and data locations of Figure 2 will be influenced by technological considerations outside our control. Managing communications for cost control and responsiveness is likely to dominate. Each transmission needs to be long enough to overwhelm the overhead for a zerolength message. Apart from this consideration, it should combine only information with good anticipated contiguity of reference. For today's workstations and telephone services, responses are likely to be from $10^{2}$ to $10^{4}$ bytes long, i.e., take from 1 to 100 seconds to deliver on a 1200-baud link. For wideband links, broadcast media, or diskette delivery, different message sizes may be better.

The interface between a presentation manager and response generators is necessarily serial, and is likely to consist of relatively short requests that are answered by longer responses generated in the data server. A workstation initiates each event, with some data server responsive as a slave. Each request (implicitly) identifies its source and encodes the selection of a response generator and the variable input needed by this response generator.

Some responses may be needed repeatedly. For example, the top of a menu hierarchy is certain to be reused and interest may focus on a subset of the lower nodes. Then each workstation can improve responsiveness, availability, and economy by saving some of the information received. If we store responses in a demand-loaded cache, each workstation will tend to accumulate just the data subset it requires. The size of each cache may be independently chosen to balance cost and responsiveness; the implementation can be hierarchical, with a semiconductor portion backed up by a disk portion. Such caches can be transferred from one workstation to another, or temporarily demounted. A single cache can serve clustered workstations. The price of all this flexibility is additional mechanism to reflect source updates in each cache. 


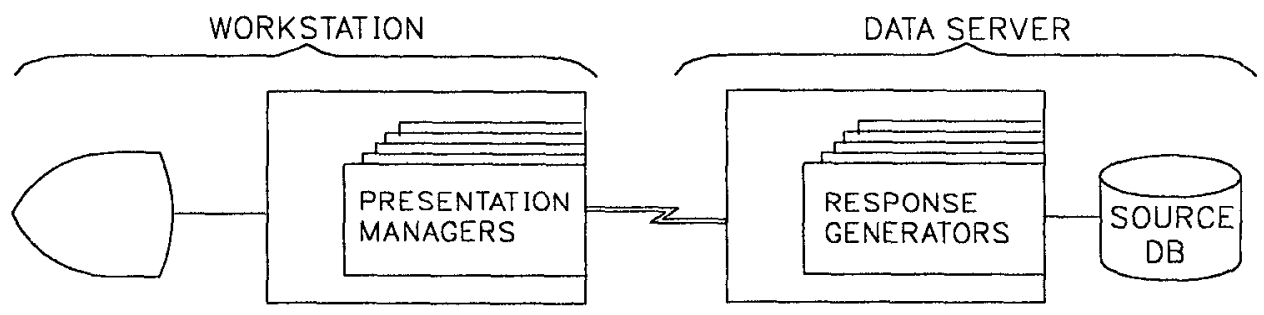

Fig. 2. Partitioned configuration for a single user.

\subsection{A Sketch of the Solution}

A trivial algorithm could maintain records derived from a source databasewhenever any change occurred in source data, every derived record would be replaced from the revised source. This would be impossibly costly, slow, and susceptible to link failures. What follows may be regarded as little more than an optimization.

The major objective is to avoid replacing any saved record that is unchanged. This is done by identifying a candidate set for the changed record set and by comparing "before" and "after" values of each candidate. Such a calculation is itself costly, and is minimized by identifying the candidate set as narrowly as possible. A scheme of hash codes on query predicates identifies which source records are changed. A "where used" or dependency relation maps candidate records in the source data space to candidate records in the derived record space.

Since workstations may be intermittently attached, change management must be split into preparation of change lists and later transmission of lists to workstations. We first describe how changes are sent to workstations, and then how change lists are prepared.

The channel between each workstation and data server can be viewed as a query interface. We refer to each query in this channel as a request to distinguish it from queries at other interfaces, and to each result in this channel as a response.

Assume that the application program in each workstation can be partitioned into a process that maintains no history (the querying process in Figure 3, implementing the logic of the presentation manager programs), and a storage component (the workstation storage manager). Typically the querying process directs information requests towards the source data and organizes responses for presentation. The storage manager is interposed in the request/response path as part of a delivery subsystem. It responds from its own resources if possible, forwards requests it cannot answer, and saves some of what it sees for future use. In the data server, a responding process constructs answers that are functions of the source database state, the request value, and how up-to-date the workstation is; the previous response generators are programs that define the logic implemented by the responding process. Responses are replicas of transforms of the source database. A replica location is any store containing data derived from a source database and required to reflect changes in that source. The obsolescence manager is a server process that filters requests and manages response consistency. 


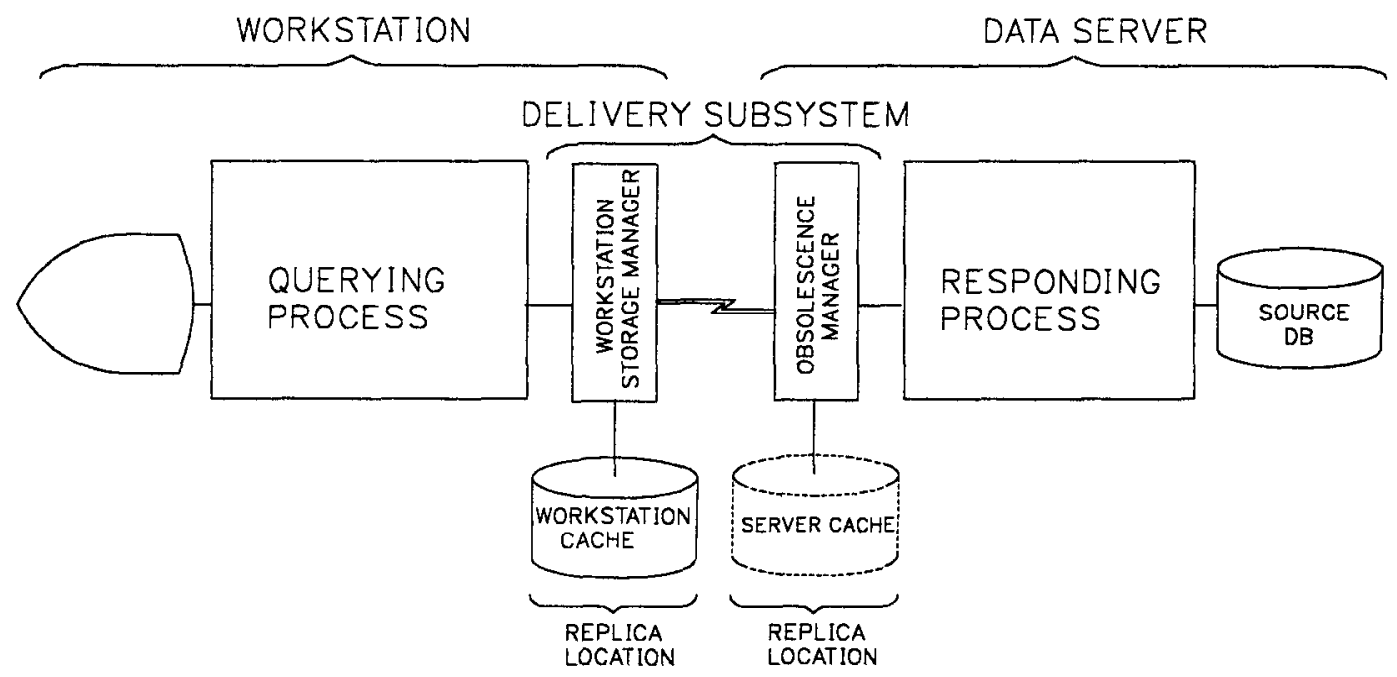

Fig. 3. Model of a single application session.

We manage the storage in this replica location as a demand-loaded cache. Each response may be saved using the corresponding request as its retrieval key. Changes in source records are managed by dropping impacted cache records; replacement is managed by the demand-loading feature. We call this process invalidation (of obsolete responses).

Several modules cooperate to ensure that a replica location proceeds from consistent state to consistent state. The querying process indicates read transaction boundaries to the workstation storage manager. The update procedures for source data define consistency by transaction boundaries and inform an obsolescence manager about changes made as part of each transaction. This obsolescence manager combines information about changes with information about the source-to-replica mapping to calculate consistency sets in the replica domain; these are represented as sets of requests. Finally, a response invalidation protocol ensures that consistency sets are applied in order and that each set is either applied as a unit or deferred for a later attempt.

A calculation is needed to identify responses impacted by a source data change. In a trivial case, each response is derived from a single source record and changes to different source records are unrelated. Then each source change requires that at most one cache record be changed and this change need not be synchronized with other changes. More generally, data from a source record is used in several replicas and source records are interrelated. We describe below one method of ensuring that the querying process becomes aware of each set of related changes all at once.

We define a consistency set to be a set of records that must be replaced as a unit if each user is to see only information corresponding to an internally valid state of the source database. A source consistency set is a subset of the records of the source database. An editor is a program (or process built on such a program) that changes a set of source database records. Each editor defines transitions 


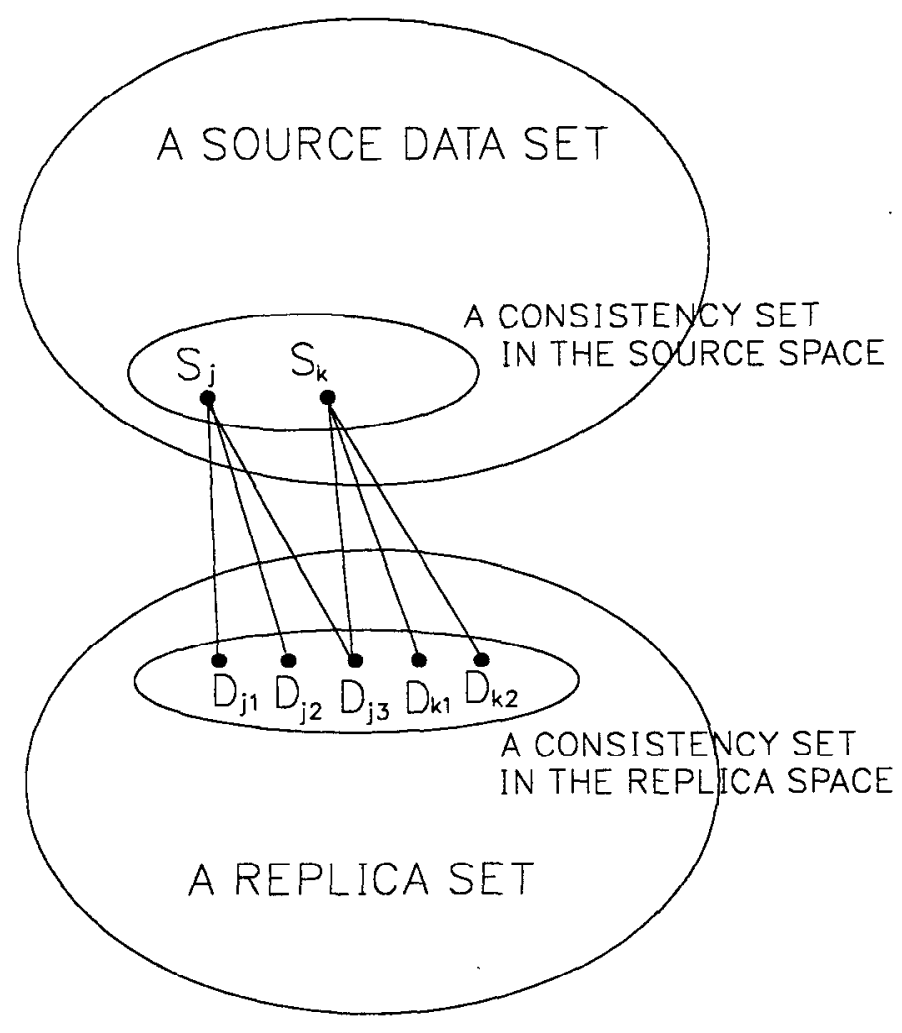

Fig. 4. Propagation of consistency.

between valid source states by a transaction discipline, or by some equivalent method; the set of records changed within a transaction is a source consistency set. A response consistency set is a set of pairs, with each first member being a request and each second member being a response. (The collection of responses in the replica space is not a set; two responses may have identical values.) A request consistency set is the set of first members in the corresponding response consistency set. We compute consistency sets in the replica record space by a mapping from consistency sets in the source record space.

Figure 4 illustrates this. A source record $\mathbf{S}_{j}$ may be used to construct several replica records $\mathbf{D}_{j 1}, \mathbf{D}_{j 2}, \ldots$. An editor may require that changes to several source records $\mathbf{S}_{j}, \mathbf{S}_{k}, \ldots$ be reflected atomically in each cache. For such an update, $\left\langle\mathbf{S}_{j}, \mathbf{S}_{k}, \ldots\right\rangle$ is the consistency set in the source domain, and $\left\langle\mathbf{D}_{j 1}, \mathbf{D}_{j 2}, \ldots, \mathbf{D}_{k 1}\right.$, $\left.\mathbf{D}_{k 2}, \ldots\right)$ is the consistency set in the replica domain.

We acquire the needed mapping by recording which portion of the source data is needed for each response built. When we change source records, this dependency relation identifies which prior responses may be obsolete. The dependency relationship relates query predicates to requests and is independent of source data versions. Query predicates are used rather than functions of query results to avoid overlooking data insertions. 
A key trick is to use the variable part of a query as the request, as the response identifier, and also as the key for locating a response in a cache. The method is economical because invalidation of obsolete responses is managed by manipulating requests.

\section{AN ALGORITHM FOR REPLICA VERSION MANAGEMENT}

In this section we describe a system with a single workstation and a single data server.

Let $\mathbf{Q}$ denote a request originating in the workstation's querying process; in addition to those queries needed to construct screen presentations, the set $\{\mathbf{Q}\}$ includes a member $\mathbf{U}$ that denotes "transaction boundary." Each request passing from the delivery subsystem to the responding process is a pair $\left(\mathbf{t}_{W S}, \mathbf{Q}\right)$. $\mathbf{t}_{W S}$ is a timestamp that indicates how up-to-date the workstation cache is; how $\mathbf{t}_{W S}$ is calculated is shown below.

Each response $\mathbf{R}$ is a function of the request and of the source database state $\mathbf{D B} \equiv \mathbf{D}(\mathbf{t})$. Apart from a constraint described in the next paragraph, this function can be whatever suits the application, i.e., cached records are arbitrary functions of source records. To support change management, the responding process must provide additional information $\mathbf{H}$ about the source database. $\mathbf{H}$ is specified below and is not a function of the database state. Thus the responding process realizes a function $G\left(t_{W S}, Q, D B\right)$ whose value is a pair $(\mathbf{R}, \mathbf{H})$.

The responding process must create a database history in the following sense. Suppose that $(\mathbf{R}, \mathbf{H})=\mathbf{G}\left(\mathbf{t}_{w s}, \mathbf{Q}, \mathbf{D}(\mathbf{t})\right)$ and $\left(\mathbf{R}^{\prime}, \mathbf{H}\right)=\mathbf{G}\left(\mathbf{t}_{w s}, \mathbf{Q}, \mathbf{D}\left(\mathbf{t}^{\prime}\right)\right)$ are two values of the response function. Then we require that $\mathbf{R}=\mathbf{R}^{\prime}$ for all $\mathbf{t}, \mathbf{t}^{\prime}$ in $\left\{t \mid t \geq t_{W S}\right\}$. This expresses that the $t_{W S}$ version of a response depends only on database contents associated with times prior to $t_{w S}$. In the following time line, with $\mathbf{t}_{u}$ and $\mathbf{t}_{v}$ as the times of source data updates, queries at $\mathbf{t}$ and $\mathbf{t}^{\prime}$ reflect neither the new information from $\mathbf{t}_{u}$ nor that from $\mathbf{t}_{v}$.

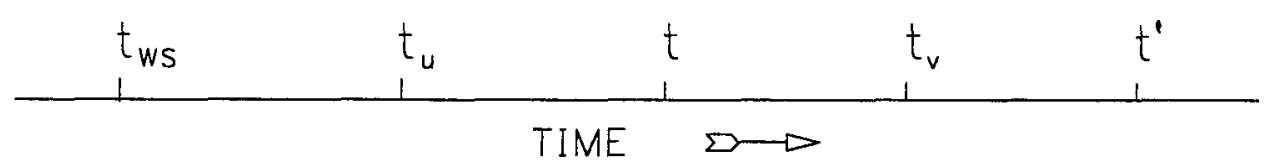

How this constraint is realized is an implementation issue outside the model. In our prototype, each source record includes a timestamp; record versions that must be grouped for consistent data views share a common timestamp. Timestamps of new records equal or exceed the time of insertion. Data updates are represented by insertions of records whose keys are identical to the outdated versions (except for the timestamp). Data deletions are represented by insertions with nulls in nonkey fields. Query subroutines limit the selected records to those with timestamps less than $\mathbf{t}_{W S}$.

\subsection{Calculating the Impact of a Source Change}

The program for a responding process may contain several database queries, which we enumerate with $\mathbf{i}=\mathbf{1}, \mathbf{2}, \mathbf{3}, \ldots$. Suppose that the ith database query in $\mathbf{G}\left(\mathbf{t}_{W S}, \mathbf{Q}, \mathbf{D}(\mathbf{t})\right)$ is constrained by the predicate $\mathbf{P}_{i}\left(\mathbf{r}, \mathbf{f}_{i}(\mathbf{Q})\right)$. Here, $\mathbf{P}_{i}\left(\mathbf{r}, \mathbf{f}_{i}(\mathbf{Q})\right)$ 
is a predicate on a source record $\mathbf{r}$ and some function $\mathbf{f}_{i}(\mathbf{Q})$ of the query value $\mathbf{Q}$. For example in a department store application the request $\mathbf{q}=$ 'TOYS $\$ 10.00$ ' might cause the responding process to invoke several queries, including a fifth one which, after substitution, is

$\begin{array}{ll}\text { SELECT } & \text { object } \\ \text { FROM } & \text { departments } \\ \text { WHERE } & \text { dept }=\text { 'TOYS' } \\ \text { AND } & \text { value }<\$ 10.00\end{array}$

In this case, we have $\mathbf{f}_{5}(\mathbf{q}) \equiv$ (FROM departments WHERE dept $=\operatorname{word}(q, 1)$ AND value $<\operatorname{word}(q, 2)$ ). Let $\mathbf{p}_{i}$ be an (application-programmer-selected) predicate over source database records $\mathbf{r}$ that selects a superset of the records selected by $\mathbf{P}_{i}$, i.e., $\mathbf{P}_{i}\left(\mathbf{r}, \mathbf{f}_{i}(\mathbf{Q})\right) \Rightarrow \mathbf{p}_{i}(\mathbf{r}, \mathbf{Q})$. $\mathbf{p}_{i}$ should not be a function of $\mathbf{t}_{W S}$ or of $\mathbf{t}$, and should not include predicate phrases with inequality operators. (This latter restriction can be overcome.) The more closely $\mathbf{p}_{i}$ approximates the complete predicate the more efficient subsequent calculations will be. In the example, a possible choice is $\mathbf{p}_{5}(\mathbf{r}, \mathbf{q})=$ (FROM departments WHERE dept $=$ 'TOYS'), which selects all the objects in the toy department rather than only the inexpensive ones.

We could store the predicate function $\mathbf{p}_{i}(\mathbf{r}, \mathbf{q})$ in the obsolescence manager. It is more convenient and economical to store a hash value from which the predicate function can be reconstructed; we choose this to be $\mathbf{h}_{i}$, a combination of a query identifier and the part of $\mathbf{q}$ used-call this string $\mathbf{s}$. For the example of the previous paragraph, we choose $\mathbf{h}_{5}=$ [5, 'TOYS']. Note that, because of the equality constraint in the choice of $\mathbf{p}_{i}$, one can find $\mathbf{s}$ in known fields of any record $\mathbf{r}$ selected by $\mathbf{p}_{i}(\mathbf{r}, \mathbf{q})$. We collect these codes into a set $\mathbf{H}(\mathbf{Q})$, which identifies a superset of all source records-past, present, and future recordsthat could be used in $\mathbf{G}$ to respond to $\mathbf{Q}$. Where $\mathbf{h}_{i} \equiv \operatorname{hash}\left(\mathbf{p}_{i}(\mathbf{r}, \mathbf{Q})\right)$, the hash set $\mathbf{H}(\mathbf{Q})$ is $\left\{\mathbf{h}_{i}(\mathbf{Q}) \mid \mathbf{i}\right.$ is a query in the response generator $\left.\mathbf{G}\right\}$. The obsolescence manager filters communications to/from the responding process to intercept $\mathbf{Q}$ and $\mathbf{H}$ and builds a binary relation $\mathbf{W} \equiv\left\{\mathbf{Q}, \mathbf{h}_{i}(\mathbf{Q})\right\}$, a "where used" table that records the dependency of responses on source subsets.

When a source editor alters $\mathbf{r}$, it also calculates each hash code $\mathbf{h}_{i}$, where $\mathbf{i}$ ranges over the unique $\mathbf{p}_{i}$. (In practical situations this is likely to be a small set.) Transaction boundaries in the editor execution define record changes that convert source data from one consistent state to another; the collected $\mathbf{h}_{i}$ for these records constitute a consistency set in the source space (see Figure 4 ). With $\mathbf{W}$, we can map these $h_{i}$ into a candidate set that identifies which responses might be impacted by the source change.

This candidate set is a set of requests; the corresponding responses are available from the responding process or in an optional data server cache (see Figure 3). The old and the new values of each response are compared; if they are different, the request is included in a replica consistency set. This set is saved until called for by a dissemination step.

\subsection{A Second Method of Calculating the Impact of a Source Change}

The method just described requires code additions to response generators and to database editors. The additions are generally small-a statement or two in response generators for each hash value and an evaluation of $\left\{\mathbf{p}_{i}(\mathbf{r})\right\}$ for each 
changed $\mathbf{r}$ in editors. This may be acceptable for new programs, but is undesirable for existing database extraction programs whose results are going to be cached.

Such invasion of application programs can be avoided if two conditions are met. A rule relating each query to the source subset needed to construct the corresponding response must be known. There must also be a means to identify all source records that have changed in a specified time interval. These facts are used in the following algorithm.

$$
\text { Let } \mathbb{P} \equiv \bigcup_{i} \mathbb{P}_{i} \text { where } \mathbb{P}_{i} \equiv\left\{\mathbf{r} \mid \mathbf{r} \in \mathbf{D B} \& \mathbf{P}_{i}\left(\mathbf{r}, \mathbf{f}_{i}(\mathbf{Q})\right)\right\} \text {. }
$$

$\mathbb{P}$ is a superset of the set of all records eligible for constructing the response to the query $\mathbf{Q}$. The $\mathbf{P}_{i}(\mathbf{r}, \mathbf{s})$ may be found by inspecting response generators. If some record $\mathbf{r}_{j} \in \mathbf{D B}$ is changed and if $\exists \mathbf{i} \exists \mathbf{P}_{i}\left(\mathbf{r}_{j}, \mathbf{v}_{i}\right)$, then each solution $\mathbf{Q}_{j}$ of $\mathbf{v}_{i}=\mathbf{f}_{i}\left(\mathbf{Q}_{j}\right)$ identifies a response that might be changed. These are collected for all $\mathbf{r}_{j}$ changed within a source edit transaction. The set

$$
\mathbb{Q}_{v}=\left\{\mathbf{Q}_{j} \mid \exists \mathbf{i} \ni \mathbf{P}_{i}\left(\mathbf{r}_{j}, \mathbf{v}_{i}\right) \& \mathbf{r}_{j} \text { is changed }\right\}
$$

contains the requests for all changed replicas.

$\mathbb{Q}_{v}$ may not be finite. Where $\mathbb{Q}_{a}$ is the set of all requests that have been answered, $\mathbb{Q}_{v} \cap \mathbb{Q}_{a}$ is finite and identifies all responses that might be changedthe candidate set identified in the prior section. This candidate set is winnowed as already described to create a request consistency set.

\subsection{Disseminating the Impact of a Source Change}

Workstation Cache. The workstation contains a storage manager WC with a store $\mathbb{C} \equiv\{(\mathbf{Q}, \mathbf{R})\}$ mapping requests to responses. The preferred WC implementation is a least-recently-used cache to get automatic, application-independent storage management. If a data change deletes all access paths to a pair $(\mathbf{q}, \mathbf{r}) \in \mathbb{C}$, this object will eventually be purged when space is needed. Garbage collection is not needed.

WC buffers the querying process from the network. Given a request $\mathbf{Q}$, the workstation storage manager returns $R$ from $\mathbb{C}$ if $(\mathbf{Q}, \mathbf{R}) \in \mathbb{C}$. Otherwise it sends $\mathbf{Q}$ to the data server. If the request to the data server succeeds, the storage manager receives $\mathbf{R}$, returns this value to the querying process and optionally stores $(\mathbf{Q}, \mathbf{R})$ in $\mathbb{C}$. If there is a communications or server failure and no response is received, WC returns a distinguished value indicating "I can't answer that now."

When the querying process communicates a transaction boundary $U$, the workstation storage manager sends $\mathbf{U}$ to the data server; the data server's obsolescence manager interprets $U$ as a cache invalidation request, i.e., the query “Which responses are obsolete?" Its reply is a request consistency set $\mathbb{Q}$. The workstation storage manager drops from the store $\mathbb{C}$ every object $(\mathbf{Q}, \mathbf{R})$ corresponding to a member of $\mathbb{Q}$ and acknowledges successful completion to the data server. The workstation storage manager itself neither needs nor implements the notion of a consistency set.

Obsolescence Manager. In addition to an optional cache, the data server includes logic and storage to manage obsolete replicas. This obsolescence manager stores sets identifying obsolete responses that are not yet removed from WC. 
Each set is delivered when the workstation indicates it is ready. Readiness is signalled by the request $\mathbf{U}$ mentioned above.

The obsolescence manager maintains a request set $\mathbb{Q}_{a}$ identifying all responses sent (needed in 3.2) and a relation $\mathbf{B}$ identifying obsolete replicas. Each element of $\mathbf{B}$ is a pair $\left(\mathbf{Q}, \mathbf{t}_{\mathrm{c}}\right)$, indicating that the response corresponding to request $\mathbf{Q}$ is obsolete. $t_{c}$ binds related updates into consistency sets and orders these sets.

At the first workstation request, the obsolescence manager adds the sentinel element $(N U L L, \infty)$ to $\mathbf{B}$. Whenever a change impact calculation at time $\mathbf{t}_{c}$ identifies a consistency set of obsolete responses $\left\{\mathbf{q}_{k}\right\}$, an element $\left(\mathbf{q}_{k}, \mathbf{t}_{c}\right)$ is added to $\mathbf{B}$ for each $\mathbf{q}_{k}$ in $\left\{\mathbf{q}_{k}\right\}$. Then the relation $\mathbf{B}$ defines the currency of the workstation $\mathbf{t}_{W S}$ to be the smaller of the current time $\mathbf{t}$ and the least timestamp in $\mathbf{B}$, viz.

$$
\mathbf{t}_{W S}=\min \left(\mathbf{t}, \mathbf{t}_{c} \ni\left[\exists\left(\mathbf{q}, \mathbf{t}_{c}\right) \in \mathbf{B} \& \neg\left(\exists \mathbf{t}^{\prime}<\mathbf{t}_{c} \ni\left(\mathbf{q}^{\prime}, \mathbf{t}^{\prime}\right) \in \mathbf{B}\right)\right]\right) .
$$

Given a request $U$ about obsolete responses, the obsolescence manager returns the earliest query consistency set in $\mathbf{B}$, viz., $\left\{\mathbf{q} \mid(\mathbf{t}, \mathbf{q}) \in \mathbf{B} \& \mathbf{t}=\mathbf{t}_{w S}\right\}$, and drops this set when the workstation acknowledges the message.

\section{AN APPLICATION-PUBLIC ACCESS INFORMATION SYSTEMS}

New opportunities for electronic information delivery [8] to a large public stimulated this work. Consider serving amateur gardeners [18, 19]. For tools, pesticides, fertilizers, flowers, shrubs, trees, etc., people need help to select items, determine planting times and conditions, evaluate hazards, determine prices and availability, etc. Computer-aided presentation permits queries that are impractical in printed catalogs, timely dissemination of dated information, and responses tailored for particular client sets. A combination of video and encoded data is needed. For example, to support diagnosis of an affliction of African violets, pictures of unhealthy plants are shown [13]; the index of a selected picture locates text about the disease and remedies. The characteristics of photographsdata volume, display rate, durability, pertinence to different applications, and cost of preparation-make local videodisk a good medium. However text and index tables are better maintained in a single location.

\subsection{Application Requirements}

This application is a prototype for diverse public information systems, e.g., city guides with maps, public transportation schedules, entertainment notices, and electronic education. Typically, many workstations will access a data server. These workstations may be close enough to each other for a local area network, or spread across an entire continent. The size of a network will range from half a dozen to thousands of workstations.

Communications will mostly move information from the data server to a workstation. Each information request will be encoded as a short string, and responses will tend to be long enough for the variable part of a single screen of alphanumeric or graphic data, i.e., 500 to 10,000 bytes. From time to time, the source database will be updated; such updates will usually be small, so that in the course of a day, only a small fraction of the database is changed. 
For such applications, we require an information dissemination subsystem with at least the following characteristics:

- Compatibility with an arbitrary mixture of DBMS technologies, but avoiding the need for a "full function" DBMS at any workstation.

- Centralized control of each source database, so that its owner can impose arbitrary limitations on what is disseminated in order to recoup his or her investment and protect his or her reputation for quality. A logically single data server is also likely to incur lower administrative costs than alternatives.

-Independent control by the owner of each workstation of data access costs, not paying for data items or telecommunications events that do not benefit him or her.

-0.1-second response times for most full-screen displays.

-Workability with slow, intermittent, and unreliable telecommunications facilities, or with mailed updates if electronic connections are unavailable or uneconomic.

-Consistent data views at any workstation, with fail-safe recovery from failures in data servers and communications links.

-Graceful scale-up to networks with thousands of workstations, workstation groups around data concentrators, and access to several data servers from any workstation.

\subsection{A Prototype}

Our prototype was part of an experimental public access information system [13], implemented with IBM PC workstations programmed with Pascal within PC-DOS and an SQL/DS [7] data server running on an IBM 370/4331. Workstations dialed to the host over 1200-baud telephone links. The communications subsystem delivered each elementary message either correctly or not at all. It was up to the new subsystem described below to recover from any residual failures and to leave the database in a consistent state if any hardware or software component terminated abnormally.

The applications were sales catalogue replacements, with video screens for pictures and alphanumeric screens representing selections in a search hierarchy (e.g., evergreen trees), descriptions of individual items (e.g., blue fir), and search on product attributes, e.g., "a 3-foot shrub that produces blue blossoms in the spring, tolerates poor drainage, ...." A typical request was 'iplant435', in which the ' $i$ ' selected a particular response generator and the 'plant435' was the variable part of an SQL SELECT statement. The response was the variable data for a screen to show the name and description of, e.g., blue delphiniums.

Support in the Data Server. We extended the system depicted in Figure 2 by a workstation and a server cache, by timestamps in source records to represent data versions, by a version manager which filters messages, and by an obsolescence manager with a private database (see Figure 5) that includes:

-a table (HASHCODE, REQUEST) relating each request REQUEST to hash identifiers of source record sets, and 


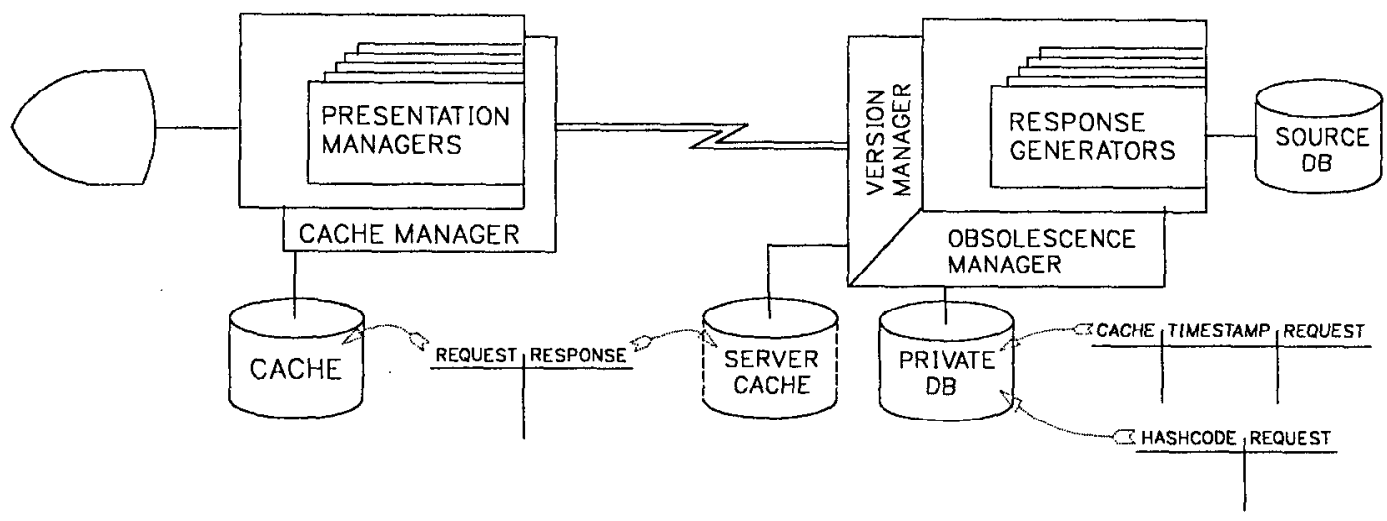

Fig. 5. Prototype configuration.

-a table (CACHE, TIMESTAMP, REQUEST) saving requests to be sent to each workstation CACHE for invalidation. Identical timestamps TIMESTAMP group requests into consistency sets and indicate the order of application. This table implicitly indicates how current each workstation is; this is the timestamp $\mathbf{t}_{W S}$ defined above.

When a request is received, the version manager informs the obsolescence manager of the identity WS of the requesting workstation and is supplied $\mathbf{t}_{W S}$, indicating how current this workstation is. This timestamp is passed, along with the request, to the response generator, which constructs its response from the latest pertinent information prior to this time. To inform the obsolescence manager which portion of the source was used to construct the response, the version manager also returns a hash code identifying each SQL query it performs.

Updating the source data is effected by an editor not depicted in Figure 5. This editor passes the hash code of each deleted or inserted source record to the obsolescence manager. (To satisfy the database history constraint, deletions are managed by inserting new records with a distinguished value in some nonkey field.) For updates, the editor passes the hash codes of both the old and new record versions. It also supplies each changed record with a timestamp, using a common value for every record altered within a transaction. When all the updates needed for a consistent source change have been made, the editor again calls the obsolescence manager to inform it that a request consistency set may be computed.

The obsolescence manager calculates which responses are impacted and COMMITs this information and the source database changes as a single transaction. This impact calculation is phased to avoid protracted database locks. First the request-to-hashcode table and a work table of changed records are used to build a list of potentially changed responses. Then the old form and the new form of each response are compared and all changed request/response pairs are entered into a new list. Response invalidation orders are entered into the table that saves these until they are asked for by workstations (via the version manager). At all 
stages, timestamps identify consistency sets of invalidations. Finally, prepared changes are marked as processed by removing their entries from the work table. ${ }^{1}$

Support in Each Workstation. A cache in each workstation (see Figure 5) answers each request from its own resources if possible, forwards other requests to the data server, and saves each response using the request as a key. If its storage is exhausted, it reclaims space by dropping request/response pairs whose probability of reuse is deemed small. We used a least recently used algorithm, weighted by the relative cost ${ }^{2}$ of building each response. Responses whose reuse are known to be improbable are not cached.

What the workstation application regards as a transaction boundary $U$, the cache manager treats as a (cache) invalidation request to obtain from the data server the requests of a set of obsolete (= invalid) responses. Each corresponding request/response pair is dropped if it is present in the cache. After all such drops, the cache manager acknowledges to the data server that it has acted upon the received set. It repeats this cycle until end-user service intervenes, or until the response to an invalidation request is an empty string signifying that the cache contains no more obsolete responses.

In the prototype, a transaction boundary $\mathbf{U}$ is issued whenever a long pause (10 minutes) between keyboard inputs suggests that the latest client has departed from the workstation. $\mathbf{U}$ is the workstation application's closest equivalent to an SQL COMMIT WORK and should be issued only when a changed view would not be perceived as an inconsistency. A COMMIT exposes to an application all database changes made since the prior COMMIT., A U does the same, except that the data view may be updated only to some past commit point if the application interrupts cache management by a data request, or not updated at all if server communications are disrupted.

Response Invalidation Protocol. Each invalidation request is handled in the data server by a specialized response generator called the version manager, which responds with the next (in timestamp order) request-consistency set for the requesting workstation. Since there may be more request strings than a single message can hold, the version manager may need to send many messages. After each message it waits for an acknowledgment; the acknowledgment is just another invalidation request. On acknowledgment of the final message of the requestconsistency set, this set is dropped from the obsolescence manager table that saves future invalidation orders. When there are no outstanding invalidation orders for the workstation, the version manager sends an empty message.

If a workstation makes some other request before the transmission of one of its request-consistency sets is complete, the version manager resets the register

\footnotetext{
${ }^{1}$ A small extension of this algorithm allows invalidation orders to be prepared out of order. For example, a retailer might wish to indicate a special offering to take effect for some bounded future period.

${ }^{2}$ A crude estimate is effective. The cost of each response is estimated by the data server and communicated in the response prefix. An alternative that adds nothing to the response generators and nothing to the response is to measure response times. The delay at the data server and the teleprocessing delay can be separately used as cost measures.
} 
that indicates how much of the set has been sent. Thus the next time the workstation requests an invalidation set, a renewed attempt will be made for this entire consistency set.

This protocol works correctly even when failures occur in message delivery. Suppose that, during processing of a request consistency set $\mathbb{R}$, a failure happens after the subset $\mathbb{R}_{1}$ of $\mathbb{R}$ has been handled, but before the complement $\mathbb{R}_{2}=$ $\mathbb{R}-\mathbb{R}_{1}$ has been handled. Since $\mathbb{R}_{1}$ is acknowledged, but $\mathbb{R}_{2}$ is not, the cache will contain no response in the set identified by $\mathbb{R}_{1}$, but may still contain obsolete versions of responses in the set identified by $\mathbb{R}_{2}$. If the workstation now requests a member of $\mathbb{R}_{1}$, the obsolete version will be transmitted from the data server, so that the cache will contain only obsolete versions of responses in $\mathbb{R}$. Whenever a renewed request for invalidations occurs, the data server will once again identify $\mathbb{R}$. No up-to-date version of any response in $\mathbb{R}$ will be transmitted until invalidations of both $\mathbb{R}_{1}$ and $\mathbb{R}_{2}$ have been acknowledged.

A price for data consistency in the workstation is occasional duplicate transmissions. How frequently this occurs depends on the statistics of the database and on reference patterns; the frequency will be very low in practical applications, as it was in executions of the prototype.

\subsection{Currency and Performance}

Sometimes users will be presented obsolete information ${ }^{3}$ and act on it. At least in retail marketing, this is already a common occurrence that is corrected administratively, if at all (e.g., for an item on sale, the aware customer gets the sale price even if the item is marked for a higher price). In fact, we would expect electronic information systems to reduce the frequency of obsolete information.

If a cache holds only a subset of the possible responses and a link to a data server is unavailable, user requests will occasionally be unanswerable. "I can't answer that now" is an acceptable response in a trade-off between availability and cost. For read-only DB access, its probability can be reduced to whatever low value is wanted.

More generally, for read-only access, a cache and remote database can be made to perform as well as a local DBMS. It is difficult to be specific without a detailed description of reference patterns and event statistics, but we can project from our prototype, which had 100,000 source records and half a dozen workstations (some emulated). The mean response length was 900 bytes and there were 5,000 potential responses for any workstation. We tested with caches of 0.8 and 3.0 Mbytes. The query complexity was such that response generation delays were comparable to transmission delays (2-20 seconds each). With the smaller cache, delays for responses were frequent; with the larger cache, our tests generated a data server request only once every several minutes. Cached responses were delivered in less than 0.1 second. We can therefore project for realistic environments that hundreds of workstations could be served by a somewhat more capable server than our 370/4331 (a 0.3 MIP machine), and that the principal activity of the central machine would be to administer updates.

\footnotetext{
${ }^{3}$ Here obsolete is to be understood in terms of timestamps. For example, if some price changes are to be effective on Monday, data are obsolete if Monday arrives and the changes have not been installed. 


\section{GENERALIZATIONS AND OPTIMIZATIONS}

For clarity, the exposition in Section 3 was framed for a single workstation and a single data server. The algorithm in fact addresses the relationship of portions of two stores, which may each be related to further stores. Our prior "source database" could itself include records derived from some other source, and the data server could include a local cache to avoid recalculations of expensive responses. Our prior "workstation" might be an intermediate node in a network hierarchy that serves many end users. We call any (portion of a) store containing records derived from an external store a replica location relative to that external store.

The prior querying process, $\boldsymbol{Q P}$, is a surrogate for a set of users-typically all users of some subsystem and typically implemented by a single application module. The prior responding process, $\boldsymbol{R P}$, is a process that answers requests by transformations of an application source database. Querying entities access source data only via responding entities. There may be several $\boldsymbol{R P}$ 's at separate geographic locations.

Each cache is associated with a single application activation. If a workstation requires several processes with independent transaction boundaries, each could have an independent workstation storage manager. Alternatively, it could use a concentrator cache as described in Section 5.1.

Messages and replica location contents as described in Section 3 are extended by identifiers of the replica and source stores involved. Each obsolescence manager manages consistency sets separately for each replica location served. Replica locations are registered automatically by obsolescence managers. Except for the small imposition described in Section 6.3, all this machinery-replica locations, storage managers, and obsolescence managers-can be buried in the delivery subsystem. Replica locations within a delivery subsystem are invisible to applications except for performance effects. Their presence improves the likelihood of prompt responses, but does not change any response. The details are available [5].

The algorithms described are economical, but can be significantly improved for large systems, such as networks with hundreds of work locations and crosscontinent spans. In this section we identify some optimizations and summarize how they are realized.

\subsection{Concentrator Caches}

When a workstation cluster is far from the source database, long distance costs may be reduced by a concentrator. A concentrator cache is a replica location whose identifier $\mathbf{I}_{C C}$ is augmented with aliases $\mathbf{I}_{C C 1}, \mathbf{I}_{C C 2}, \ldots$ which are unique among replica location identifiers $\mathbf{I}_{i}$. To a data server, a concentrator is indistinguishable from a small set of $\boldsymbol{Q P}$ 's. To a workstation, it is indistinguishable from a set of $\boldsymbol{R P}$ 's. The tables of a concentrator are similar to a cache and the table $\mathbf{B}$ in a data server, extended by a column for a responding process identifier $\mathbf{I}_{R P}$. Its space management rules are also similar.

Its logic is more complex. A concentrator cache $\mathbf{C C}$ represents workstations of varying currencies without reflecting the number of workstations by using each 
alias as a surrogate for $\boldsymbol{Q P}$ 's with a common currency. For a request $\left(\mathbf{I}_{R P}, \mathbf{I}_{Q P}, \mathbf{Q}\right)$ that cannot be satisfied by the cache, the concentrator sends $\left(\mathbf{I}_{R \Gamma}, \mathbf{I}_{C C}, \mathbf{Q}\right)$ to the server, where $\mathbf{I}_{C C j}$ corresponds to $\mathbf{t}_{Q P}$. The result $\mathbf{R}$ is stored in $\mathbf{C C}$ in the record $\left(\mathbf{t}_{Q P}, \mathbf{I}_{R P}, \mathbf{Q}, \mathbf{R}\right)$ and sent to $\boldsymbol{Q P}$. An algorithm for maintaining aliases and the obsolete replica table $B$ is available [5].

A concentrator cache may be added to or dropped from a network without change to source or workstation logic. Such caches may be cascaded.

\subsection{Workstation Differentiation}

Response values may depend on workstation locations. For example, the best time to plant tomatoes is different in San Jose and San Francisco. The simplest approach would be to include the workstation identifier as part of each request Q. However, this would make server processing loads and server cache space demands proportional to the number of workstations. Often there is a more economical solution because most information is common to all locations, e.g., for remedies for a plant disease; in the Western Garden Book [19] less than 5 percent of the information depends on location.

A natural approach to modeling differences among workstations uses workstation attributes, such as "in California" and "is a pharmacy," and a single attribute tagging each source record. The record is selected only if the workstation satisfies the tag. Among records whose keys differ only in the tag, the record with the earliest value in a tag ordering is selected. For example, suppose answers to some query are available for "in U.S.A." and for "in California," but not for "in San Francisco." Then for a workstation declared to be in San Francisco, we would provide the "in California" version of the answer. (The response generator would, of course, have to implement the ordering "in U.S.A." $>$ "in California" $>$ "in San Francisco.")

Workstations with the same attributes get identical responses. Data server and concentrator caches associate entries with response equivalence classes rather than with individual workstations. This reduces cache sizes and response generator loads to proportionality to the number of equivalence classes. Accidental equalities between responses are still common, particularly for requests whose answer is independent of workstation attributes. The response generator load for these cannot be avoided, except by application specific measures, but cache space can be saved by using pointers.

The economies afforded by these techniques have been demonstrated in the prototype. To support workstation differentiation in application programs requires no change to workstation portions and only a few lines in each response generator. The changes to the delivery subsystem have been described [5].

\subsection{Cache Backup and Recovery}

The delivery subsystem minimizes the effect of communications and data server failures, but is not proof against cache failure. A faulty cache could be abandoned in favor of a new empty cache. However the cost and delay to load a large cache may be unacceptable.

The obsolescence manager's database can serve as a cache recovery log. Each workstation maintains a checkpoint cache copy with a timestamp that is updated 
after every response invalidation. This timestamp, $\mathbf{t}_{w}$, is obtained from the data server as part of the latest obsolete response set. Any update with a timestamp $\mathbf{t} \ni \mathbf{t}_{w}<\mathbf{t}<\mathbf{t}_{Q P}$ has been lost between the time of the backup cache and the damaged cache. As part of recovery, requests for the affected responses are dealt with before any other consistency set. This may occur whenever resources are available; the workstation application may proceed immediately after switching to a backup cache if some obsolete data are acceptable.

\subsection{Overlap}

Replacing obsolete records can be overlapped with service to the querying process. To avoid inconsistency in what is delivered to the querying process, access to replacement records is delayed until each change set is completely delivered and until the querying process ends a transaction. In the meantime, requests for elements of the change set are answered with obsolete responses.

\section{DISCUSSION}

The need to maintain remote copies is growing. Two cases may be distinguishedfaithful replicas and lossy ones. A faithful replication has an inverse mapping; a lossy one does not. Faithful replica maintenance is typified by file replication schemes, such as ASCII-to-EBCDIC conversion. In this case, the data do not implicitly determine which instance is the source and which is the replica. This information must be represented in explicit tables [6] or in policies imbedded in programs; the latter is common in distributed file systems, e.g. in Andrew [15].

Lossy replica management is typified by information distribution, such as the horticultural application that we have drawn on, or maintenance of compiled and linked derivations of source programs. In this case, the distinction between source and replica is clear, but information about the dependency of replicas on source objects must be provided. This can be (1) extracted from the replica generators, as in our first method of calculating the impact of a source change; (2) supplied as an explicit rule, as in our second impact calculation method; or (3) supplied in tables by some user, as is done in the system-build method described by Schmidt [17].

\subsection{Consistency and Currency of Views}

A new approach to installing database updates was needed because of quantitative aspects of potential configurations and usage patterns, and because updates cannot always be reflected promptly at workstations. For example, in a sales network with a hundred terminals, each customer session might be a transaction in which any price shown is to be unchanged from start to finish. A session might last for many minutes, could occur at any time of day, and might present prices from hundreds of database records. Suppose that the merchant wishes to lower all prices, and asks that this be consummated promptly and as a single transaction. With a locking discipline, the update cannot be posted until all customer sessions are closed. If the duty cycle of each terminal is 10 percent, then the probability that no customer is active is 0.0026 percent. The desired update is effectively blocked. We avoid this problem if we let some customers see the new prices while others still see the old ones. 
Updates must be activated in each workstation in controlled groups if the customer is to perceive consistency. The stream that constitutes a consistent unit may take some time to transmit (e.g., the description of a new automobile model). Transmission may be interrupted, either involuntarily by a failure, or purposely to reallocate the workstation's resources to prompt customer service. Until the transmission can be rescheduled and completed, obsolete data must be shown.

Consistency has to do with distinguished occasions in the course of a computation-occasions in which an editor indicates that the modified database conforms to an external notion of correctness [3]. Garcia-Molina and Wiederhold [4] have classified objectives for read-only copies. They identify three consistency levels: No consistency, in which individual query results are not guaranteed to satisfy constraints; weak consistency, in which individual results satisfy constraints; but guarantees are not made for pairs of query results; and strong consistency, in which the schedule of all transactions in a network is equivalent to a serial schedule [20]. They classify currency as: $t$-vintage currency, in which a query obtains data as they existed at a specified earlier time; $t$-bound currency, in which query results reflect all updates committed up to a specific time; and no currency requirement.

Our method is $t$-bound, and seems to be somewhat stronger than weakly consistent. (Garcia-Molina is not entirely clear on read-only transaction boundaries.) Editors use transactions to indicate collections of data changes that are transitions between valid database states. Each reader's data view reflects only complete update transactions. ${ }^{4}$ The data view within a read transaction is consistent for some point in time, which may be a past instant in the history of the source database. Read transactions at different workstations are not synchronized; someone who could simultaneously see a pair of workstations might observe different answers to the same query. The algorithm is different than the closest Garcia-Molina suggestion (their TCA [4, p. 227]) to allow (a) consistency within a sequence of queries; (b) incomplete replicas held in workstations; (c) robustness against network failures during replica delivery; and (d) replicas that are arbitrary transformations from source data.

\subsection{Pervasiveness}

The obsolescence-management mechanism is intended for data with low volatility, measured as the fraction of the source records that change in any time period. One might be concerned that performance would limit the approach to a narrow application set; this does not seem to be the case. Table I projects some data size and data change rates, showing that there are many important applications for which the machine load and communications traffic are not practical or economic barriers. The prototype has demonstrated change rates an order-of-magnitude larger than the projections.

The algorithm for maintaining local copies relies on good data partitioning to achieve good network performance. The examples suggest that many applications naturally have favorable reuse statistics. A more convincing argument is based

${ }^{4}$ Consistent copy, as defined by Malhotra [12], is similar. 
Table I. Load Estimates for Data Changes

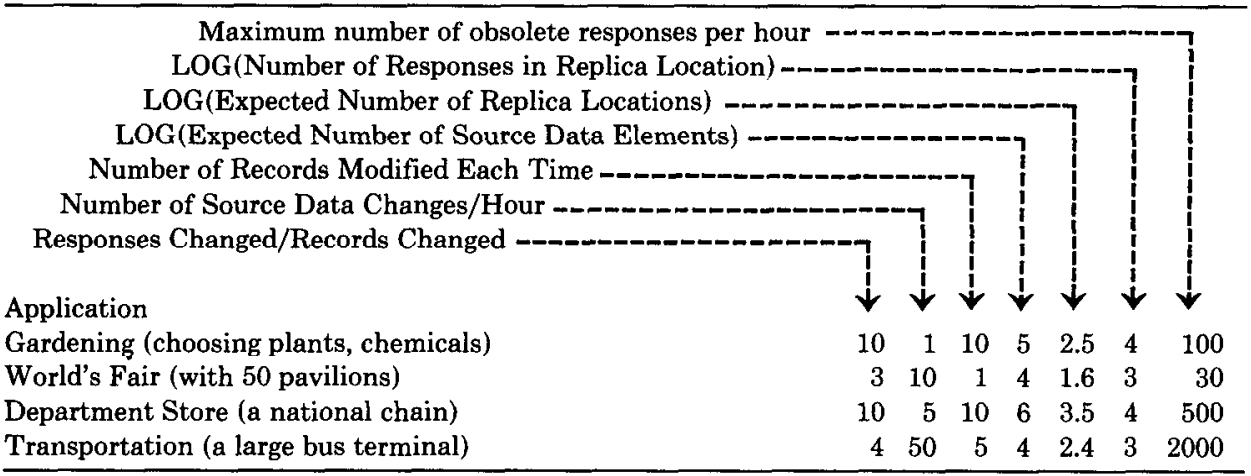

on what is likely to make end users effective, and on the likely objectives of information providers.

HYPOTHESIS 1. When individuals seek information, they are most effective and most satisfied if each presentation frame embeds a relatively small amount of new data in a familiar framework. ${ }^{5}$

Here we contrast what is suitable for information media with what is appropriate for entertainment or cultural media. In cultural media, variable contexts and colorations are important as matrices that convey themes indirectly-their importance may even exceed that of story threads, as in Charles Dickens' or Joseph Conrad's writings, in which the personality of a hero is conveyed by his behavior in a milieu. In contrast, familiar contexts in information services speed subscribers to small numbers of pertinent facts. Application designers frequently act implicitly on this hypothesis.

HyPOTHESIS 2. The objective of each information provider is to present similar data to each of a large number of individual subscribers.

This kind of reuse contributes to economies of scale that give competitive advantage.

We conclude that our method for management of distributed data is widely useful since it is grounded in common human needs, rather than the specifics of any application class. How extracts from data collections are combined into presentation frames will depend on circumstances and on applicationprogrammer styles. If other design considerations do not lead to responses having a high probability of reuse, designers should reorganize the implementation. Heuristic guidelines ${ }^{6}$ are readily given.

\footnotetext{
${ }^{5}$ Here the framework can include screen format data, constant headers, colors, icons, assignments of programmable function keys, etc. In a newspaper, it can include position in the edition, position on a page, type-font, size and wording of title, etc.

${ }^{6}$ Both the hypotheses and the exploitation of their consequences are similar to how contiguity of reference underlies paging mechanisms in virtual storage systems. Specifically, in most programs there is enough contiguity of reference that no special action is necessary; however, programmers are advised of simple techniques to improve paging statistics.
} 


\subsection{Application Program Responsibilities}

Most of an implementation can be hidden in the communications subsystem. However there seems to be an unavoidable burden on application programmers. In order to support an arbitrary relationship between source data schema and the content of responses, the method requires the authors of response generators and of source-data editors to communicate "where used" information. We argue first that this is a reasonable burden and then that it is generally feasible to make a good choice.

Source data edit programs must use the "where used" scheme defined by response generators, i.e., the author of an editor must know the queries used and the related hash code scheme. This is akin to the knowledge (s)he needs about table schema, and can be considered to be an extension of each schema that has to be communicated as part of an application description. No communication is needed when the authors of editors are the same individuals as the authors of response generators.

It is always possible to choose "where used" codes so that no affected response is overlooked when a source record is changed. A correct, but inefficient choice is to indicate all source tables that have changes. The most efficient choice minimizes the source subset depicted in Figure 4. The trick is to use part of the predicate of each query in the response generation program. In the query

$\begin{array}{ll}\text { SELECT } & \text { some fields } \\ \text { FROM } & \text { SOMETABLE } \\ \text { WHERE } & \text { field1 = :value1 } \\ \text { AND } & \text { field2 = :value2 } \\ \text { AND } & \cdots\end{array}$

we might choose value1, prefixed by a code to identify SOMETABLE, as the hash code. If each editor communicates the content of field1 for each deleted, altered, or inserted record, then the obsolescence manager will find all (potentially) impacted responses. Alternatively, we might choose field2 or a function of both fields. The last alternative is likely to make the impact calculation more efficient than the former two.

A record schema may have more than one hash code defined; this is needed if different queries use predicates on nonintersecting sets of fields. Multiple hash codes tend to make the impact calculation consider a larger set of records.

\subsection{Comparison with Distributed DBMS Work}

The closest earlier technology is general-purpose, distributed database management systems. In reviewing these, Mohan [14] notes that "While numerous algorithms have been proposed for performing updates to replicated data, strangely enough there does not exist, to my knowledge, an efficient and reliable implementation of any of those algorithms in a general-purpose DBMS system."

The use of replicas for performance and availability of read-only data is not, in itself, new. Systems that support read-only replica mechanisms include Mermaid [21], Multibase [9], SDD-1 [16], and R* [11]. Traiger [20, p. 331] considers a mechanism similar to part of our obsolescence manager to batch updates within a normal locking protocol. In Table II and the next paragraph we focus for comparison on $\mathrm{R}^{*}$ and "snapshots" [10]. 
Table II. Comparison of $\mathrm{R}^{*}$ Snapshots and Cache Invalidation

\begin{tabular}{lll} 
& \multicolumn{1}{c}{$\mathrm{R}^{*}$ Snapshots } & \multicolumn{1}{c}{ Cache invalidation } \\
\hline Replica content & Arbitrary SQL view & Arbitrary DB derivation \\
Replica size & Complete SQL view & Time-varying subset \\
Site similarity & Sites equivalent & Data server(s) + workstations \\
Network size & $2-20$ nodes for a table & Potentially thousands of workstations \\
Communications & CICS virtual circuits & Serial interface \\
System software & DBMS at all locations & DBMS at data server locations \\
Transparency [20] & No & Yes \\
& \multicolumn{2}{c}{} \\
Updatc timing & \multicolumn{2}{c}{ Application controlled } \\
Restrictions & \multicolumn{2}{c}{ Read only } \\
Read consistency & By location \\
Read/write consistency & Application responsible \\
\hline
\end{tabular}

Adiba [1] suggests using "a snapshot [to] store the result of a single evaluation of the query for subsequent access" and "to increase the data reference locality in distributed databases by defining locally stored snapshots based on remote data values." This proposal has been implemented [10] in $R^{*}$, where a snapshot is a locally held replica of the database projection. A snapshot user is not protected against read-write conflicts [2, p. 192], but must ensure that his or her snapshot is fresh. The DBMS must be installed in each workstation. $\mathrm{R}^{*}$ was not intended for a system with hundreds of workstations, and is more expensive, in the replica locations, than the cache approach. Of course, the $\mathrm{R}^{*}$ system supports "fullfunction" database services at each node.

It seems possible to implement our style of cache as a table in a workstation relational database, with a view exposed to the application. This would make available the full power of the query language, and would also permit an easy implementation of overlapped user service and cache-update management. Such an implementation would seem to offer everything that snapshots do without restricting the content of the cache to be a projection of the source data. We have not implemented such a cache, so the suggestion is speculative.

\section{CONCLUSION}

We have described a new approach to information delivery in large, weakly connected networks. It maintains replicas in stores close to query locations and disseminates source data changes by calculating which replicas are affected, purging obsolete replicas, and fetching of up-to-date versions on application demand. It can be regarded as a specialized distributed DBMS, which is of interest because, at least at the current state of technology, general-purpose DBMS's are not economical for modestly priced workstations.

The method is robust under failures of processors, processes, and communication links. It minimizes communications traffic, provides user service when communications are not available, and minimizes incremental costs in workstations. Update in remote workstations is handled by a system component that is transparent to the rest of the workstation implementation and is, apart from program linkage protocols, independent of the workstation and data-server technologies used to implement the application. 
While our prototype accesses source data with an SQL DBMS, the architecture described is more general, extending to source data in a mixture of file systems, DBMS-accessed databases, and other storage subsystems. While the estimated requirements originated in models of public information services, the area of application is thought to be more pervasive.

\section{ACKNOWLEDGMENTS}

A. Peled suggested public information as an area of investigation. R. L. Mattson designed the retail-sales-support system and suggested the mainfold benefits of caching application data in workstations, $D$. J. Lorch implemented the retailsales application and the cache manager, and participated in designing the communications protocol for invalidating cache contents. Discussions with these colleagues and members of the San Jose $R^{*}$ project are gratefully acknowledged.

Comments by Paul Wilms and by the referees of an early manuscript have helped the author towards the current version; their time and care is gratefully acknowledged.

\section{REFERENCES}

1. Adiba, M. E., AND LindSAY, B. G. Database Snapshots, IBM Research Report RJ2772, 1980.

2. Bernstein, P. A., AND Goodman, N. Concurrency control in distributed database systems. Comput. Surv. 13, 1 (1981), 185-221.

3. Eswaran, K. P., Gray, J. N., Lorie, R. A., and Traiger, I. L. The notions of consistency and predicate locks in a database system. Commun. ACM 19, 11 (1976), 624-633.

4. Garcia-Molina, H., aND Wiederhold, G. Read-only transactions in a distributed database. ACM Trans. Database Syst. 7, 2 (1982), 209-304.

5. Gladney, H. M. A model for distributed information networks. IBM Res. Rep. RJ5220, 1986. Available from the librarian at the IBM Almaden Research Center.

6. Gladney, H. M., Artsy, Y., AND Chang, H. Y. Note: File synchronization in weakly coupled networks. IBM Res. Rep. RJ5043, 1985.

7. INTERnational Business Machines. SQL/Data System. IBM Program Product 5748-XXJ, 1983.

8. Ives, B., and Learmonth, G. P. The information system as a competitive weapon. Commun. ACM 27, 12 (1984), 1193-1201.

9. LANDERS, T., AND Rosengerg, R. L. An overview of MULTIBASE. In Distributed Data Bases. Proceedings of Second International Symposium on Distributed Data Bases. H. J. Schneider, Ed., North-Holland, Amsterdam, The Netherlands, Sept. 1982.

10. Lindsay, B., HaAs, L. M., Mohan, C., Pirahesh, H., and Wilms, P. A snapshot differential refresh algorithm. IBM Res. Rep. RJ4992, 1986. Also in ACM Proceedings SIGMOD 15, 2 (1986), 53-60.

11. Lohman, G. M., Mohan, C., HaAs, L. M., Lindsay, B. G., Selinger, P. G., and Wilms, P. F. Query processing in R*, IBM Research Report RJ4272, 1984.

12. Malhotra, A., Markowitz, H. M., and Pazel, D. P. Consistent copy: A new database facility, IBM Research Report RC9630, 1982.

13. MatTson, R. L. ET AL. VIEW \& SHOP prototype, 1983-84.

14. Mohan, C. Recent and future trends in distributed data base management. IBM Res. Rep. RJ4240, 1984.

15. Morris, J. H., Satyanaranyanan, M., Conner, M. D., Howard, J. H., Rosenthal, D. S. H., AND Smith, F. D. Andrew: A distributed personal computing environment. Commun. ACM 29, 3, 184-201, 1986.

16. Rothnie, J. B., Bernstein, P. A., Fox, S., Goodman, N., Hammer, M., Landers, T. A., REEve, C., SHIPMAN, D. W., AND WONG, E. Introduction to a system for distributed databases (SDD-1), ACM Trans. Database Syst. 5, 1 (1980), 1-17. 
17. Schmidt, E. E. Controlling Large Software Development in a Distributed Environment. Ph.D. dissertation, University of California at Berkeley, 1982. Also appeared as Xerox PARC report CSL-82-7.

18. Smith, M. D., Ed. The Ortho Problem Solver. Ortho Information Services, San Francisco, 1982.

19. Sunset Magazine Editors. Western Garden Book, Lane Magazine and Book Co, Menlo Park, Cal., 1970.

20. Traiger, I. L., Gray, J. N., Galtieri, C. A., and Lindsay, B. G. Transactions and consistency in distributed database systems. ACM Trans. Database Syst. 7, 3 (1982), 323-342.

21. Yu, C. T., and Chang, C. C. On the Design of a Query Strategy in a Distributed Database Environment. ACM Proceedings of SIGMOD 15, 2 (1983), 30-39.

Received June 1986; revised February 1987 and November 1987; accepted February 1988 